\title{
A relativistic type Ibc supernova without a detected $\gamma-$ ray burst
}

\author{
A. M. Soderberg ${ }^{1}$, S. Chakraborti ${ }^{2}$, G. Pignata $^{3}$, \\ R. A. Chevalier ${ }^{4}$, P. Chandra ${ }^{5}$, A. Ray ${ }^{2}$, M. H. Wieringa ${ }^{6}$, A. Copete ${ }^{1}$, \\ V. Chaplin ${ }^{7}$, V. Connaughton ${ }^{7}$, S. D. Barthelmy ${ }^{8}$, M. F. Bietenholz ${ }^{9,10}$, \\ N. Chugai ${ }^{11}$, M. D. Stritzinger ${ }^{12,13}$, M. Hamuy ${ }^{3}$, C. Fransson ${ }^{14}$, O. Fox $^{4}$, \\ E. M. Levesque ${ }^{1,15}$, J. E. Grindlay ${ }^{1}$, P. Challis ${ }^{1}$, R. J. Foley ${ }^{1}$, R. P. Kirshner ${ }^{1}$, \\ P. A. Milne ${ }^{16}$, M. A. P. Torres ${ }^{1}$ \\ ${ }^{1}$ Harvard-Smithsonian Center for Astrophysics, 60 Garden Street, MS-51, Cambridge, MA 02138, USA \\ 2 Tata Institute of Fundamental Research, Mumbai 400 005, India \\ 3 Departamento de Astronomi'a, Universidad de Chile, Casilla 36-D, Santiago, Chile \\ ${ }^{4}$ University of Virginia, Department of Astronomy, PO Box 400325, Charlottesville, VA 22904, USA \\ ${ }^{5}$ Royal Military College of Canada, Kingston, ON Canada \\ ${ }^{6}$ Australia Telescope National Facility, CSIRO, Epping 2121, Australia \\ 7 University of Alabama, Huntsville, AL, USA \\ 8 NASA Goddard Space Flight Center, Greenbelt, MD 20771, USA \\ ${ }^{9}$ Department of Physics and Astronomy, York University, Toronto, ON M3J 1P3, Canada \\ ${ }^{10}$ Hartebeestehoek Radio Observatory, PO Box 443, Krugersdorp, 1740, South Africa \\ ${ }^{11}$ Institute of Astronomy, RAS, Pyatnitskaya 48, Moscow 119017, Russia \\ ${ }^{12}$ Las Campanas Observatory, Carnegie Observatories, Casilla 601, La Serena, Chile \\ ${ }^{13}$ Dark Cosmology Centre, Niels Bohr Institute, University of Copenhagen, Copenhagen, Denmark \\ ${ }^{14}$ Department of Astronomy, Stockholm University, AlbaNova, SE-106 91 Stockholm, Sweden \\ ${ }^{15}$ Institute for Astronomy, University of Hawaii, 2680 Woodlawn Dr., Honolulu, HI 96822, USA \\ ${ }^{16}$ Steward Observatory, University of Arizona, 933 North Cherry Avenue, Tucson, AZ 85721, USA
}

Long duration gamma-ray bursts (GRBs) $\operatorname{mark}^{1}$ the explosive death of some massive stars and are a rare sub-class of Type Ibc supernovae (SNe Ibc). They are distinguished by the production of an energetic and collimated relativistic outflow powered ${ }^{2}$ by a central engine (an accreting black hole or neutron star). Observationally, this outflow is manifested ${ }^{3}$ in the pulse of gamma-rays and a long-lived radio afterglow. To date, central engine-driven SNe have been discovered exclusively through their gamma-ray emission, yet it is expected ${ }^{4}$ that a larger population goes undetected due to limited satellite sensitivity or beaming of the collimated emission away from our line-of-sight. In this framework, 
the recovery of undetected GRBs may be possible through radio searches ${ }^{5,6}$ for SNe Ibc with relativistic outflows. Here we report the discovery of luminous radio emission from the seemingly ordinary Type Ibc SN 2009bb, which requires a substantial relativistic outflow powered by a central engine. The lack of a coincident GRB makes SN 2009bb the first engine-driven SN discovered without a detected gamma-ray signal. A comparison with our extensive radio survey of SNe Ibc reveals that the fraction harboring central engines is low, $\sim 1 \%$, measured independently from, but consistent with, the inferred ${ }^{46}$ rate of nearby GRBs. Our study demonstrates that upcoming optical and radio surveys will soon rival gamma-ray satellites in pinpointing the nearest engine-driven SNe. A similar result for a different supernova is reported ${ }^{8}$ independently.

On 2009 Mar 21.1 UT, the Chilean Automatic Supernova Search Program (CHASE; Ref. 31) discovered ${ }^{10}$ a bright optical transient through repeated imaging of the nearby spiral galaxy NGC 3278 at a distance, $d \approx 40 \mathrm{Mpc}$. The new object was offset $22 \operatorname{arcsec}$ $(4.2 \mathrm{kpc})$ from the center of the galaxy and located within its star-forming disk. Optical spectroscopy obtained on Mar 28.1 UT revealed ${ }^{11}$ that the transient was a young Type Ibc SN (hereafter SN 2009bb) lacking evidence for Hydrogen in the explosion debris. Based on the previous non-detection of SN 2009bb on Mar 19.2 UT, we tightly constrain the SN explosion date to be Mar $19 \pm 1$ UT (see Suppl. Info.).

Using the Very Large Array (VLA) on Apr 5.2 UT, we discovered a coincident radio counterpart at $\alpha(\mathrm{J} 2000)=10^{\mathrm{h}} 31^{\mathrm{m}} 33.87^{\mathrm{s}}$ and $\delta(\mathrm{J} 2000)=-39^{\circ} 57^{\prime} 30.1^{\prime \prime}( \pm 0.7 \operatorname{arcsec}$ in each coordinate) and with a flux density, $F_{\nu}=24.53 \pm 0.06 \mathrm{mJy}$, at frequency, $\nu=8.46$ GHz. This corresponds to a spectral radio luminosity of $L_{\nu} \approx 5 \times 10^{28} \mathrm{erg} \mathrm{s}^{-1} \mathrm{~Hz}^{-1}$ at $\Delta t \approx 17$ days after explosion, more luminous than any other SN Ibc observed ${ }^{5,6,12}$ on a comparable timescale. Instead, the radio properties of SN 2009bb are consistent with the sample of nearby $(z \lesssim 0.1)$ GRBs, observed to consistently yield ${ }^{46}$ lower relativistic energies than "classic" GRBs preferentially discovered at larger distances. Further VLA observations of SN 2009bb revealed a power-law flux decay, $F_{\nu, 8.46 \mathrm{GHz}} \approx t^{-1.4}$, in line with the radio afterglow evolution seen ${ }^{13}$ for the nearest gamma-ray burst, GRB 980425 at a similar distance of $d \approx 38 \mathrm{Mpc}$ (Figure 1 ). 
Unlike the optical emission from $\mathrm{SNe}$ which traces only the slowest explosion debris, radio observations uniquely probe ${ }^{35}$ the fastest ejecta as the expanding blastwave (velocity, $v$ ) shocks and accelerates electrons in amplified magnetic fields. The resulting synchrotron emission is suppressed by self-absorption (SSA) producing a low frequency radio turnover that defines the spectral peak frequency, $\nu_{p}$. Combining our observations from the VLA and the Giant Meterwave Radio Telescope (GMRT), the radio spectra of SN 2009bb are well described by an SSA model across multiple epochs (Figure 2). From our earliest spectrum on Apr $8 \mathrm{UT}$ ( $\Delta t \approx 20$ days), we infer $\nu_{p} \approx 6 \mathrm{GHz}$ and a spectral peak luminosity, $L_{\nu, p} \approx 3.6 \times 10^{28} \mathrm{erg} \mathrm{s}^{-1} \mathrm{~Hz}^{-1}$.

Making the conservative assumption that the energy of the radio emitting material is partitioned equally into accelerating electrons and amplifying magnetic fields (equipartition), the properties of the SSA radio spectrum enable ${ }^{13,35}$ a robust estimate of the blastwave radius, $R \approx 2.9 \times 10^{16}\left(L_{\nu, p} / 10^{28} \mathrm{erg} \mathrm{s}^{-1} \mathrm{~Hz}^{-1}\right)^{9 / 19}\left(\nu_{p} / 5 \mathrm{GHz}\right)^{-1} \mathrm{~cm}$. Luminous synchrotron sources with a low spectral peak frequency thus require larger sizes (Figure 3). For SN 2009bb, we infer $R \approx 4.4 \times 10^{16} \mathrm{~cm}$ at $\Delta t \approx 20$ days and thus the mean expansion velocity is $R / \Delta t=0.85 \pm 0.02 c$, where $c$ is the speed of light. The transverse expansion speed, $\Gamma \beta c=R / \Delta t$ indicates that the blastwave is relativistic, $\Gamma \gtrsim 1.3$, at this time [bulk Lorentz factor $\Gamma=\left(1-\beta^{2}\right)^{-1 / 2}$ with $\beta=v / c$ ]. This is a lower limit on the initial velocity since the radio evolution indicates that the blastwave decelerated early on. We further find that the radio emission requires a minimum energy, $E=(1.3 \pm 0.1) \times 10^{49} \mathrm{erg}$, coupled to the relativistic outflow and comparable to the values inferred ${ }^{46,13,15,16}$ from the radio afterglows of nearby GRBs (see Figure 4).

These conclusions are robust; the blastwave velocity is insensitive $\mathrm{e}^{35,37}$ to deviations from equipartition while the relativistic energy can only be higher. ${ }^{18}$ In view of these constraints, we note that shock-acceleration in some SNe Ibc may ${ }^{19}$ couple a minute fraction $(\lesssim 0.01 \%)$ of the total energy, $E_{\mathrm{tot}}$, to material with a trans-relativistic velocity. However, this scenario would require an exceedingly high total energy for SN 2009bb, $E_{\text {tot }} \gtrsim 10^{53} \mathrm{erg}$, a factor of $10^{2}$ higher than the total explosion energies inferred for SNe Ibc. We conclude that the energetic and relativistic outflow from SN 2009bb was powered by another energy reservoir, a central engine. To date, engine-driven SNe have been discovered exclusively through their 
gamma-ray emission, making SN 2009bb the first to be identified by its long-wavelength signal.

Motivated by our discovery of an engine-driven relativistic outflow, we searched for a gamma-ray counterpart in temporal and spatial coincidence with SN 2009bb. During our bracketed explosion date estimate, the all-sky Interplanetary Network (IPN; Ref. 44) of high energy satellites did not detect a coincident GRB (see Suppl. Info.). Based on the IPN sensitivity and detection efficiency, we place an upper limit on the gamma-ray fluence of $F_{\gamma}<5 \times 10^{-6} \mathrm{erg} \mathrm{cm}^{-2}$, corresponding to an energy of $E_{\gamma}<10^{48} \mathrm{erg}$ (band, 25-150 keV) if the relativistic outflow was isotropic. This limit is a factor of two higher than the isotropicequivalent $E_{\gamma}$ observed $^{45}$ from GRB 980425, and thus it is possible that SN 2009bb gave rise to a similar (albeit undetected) signal. At the same time, these limits cannot exclude scenarios in which the SN (i) powered a GRB directed away from our line-of-sight, or (ii) did not produce any gamma-rays. SN 2009bb observationally demonstrates the limitation of using gamma-ray satellites as a primary tool to identify nearby engine-driven explosions.

In this context, we note that our VLA observations of SN 2009bb were obtained as part of an extensive radio survey of 143 optically-discovered local SNe Ibc designed to recover relativistic SNe without detected gamma-ray counterparts. This systematic radio study revealed $^{46,5,6,12}$ no additional relativistic $\mathrm{SNe}$, instead indicating typical maximum blastwave velocities for SNe Ibc of $\beta \approx 0.1$ (Figures 3 and 4 ). From this sample, we therefore estimate the fraction of engine-driven SNe to be just $0.7_{-0.6}^{+1.6 \%}(1 \sigma)$. This is measured independently from, and yet consistent with, the fraction inferred from the relative rates ${ }^{46,49,50}$ of nearby GRBs and all SNe Ibc, $1.4_{-0.3}^{+5.0 \%}(1 \sigma)$. Our long-term study thus confirms that engine-driven SNe are uncommon.

The infrequency of relativistic outflows among massive star explosions implies that their progenitor stars share an essential and rare physical property. Observations of the explosion environment may offer unique clues. On a local scale $(\lesssim 1 \mathrm{pc})$, where the environment was shaped directly by the evolution of SN 2009bb's progenitor star, we find evidence for a pre-explosion mass loss rate of $\dot{M}=(2.0 \pm 0.2) \times 10^{-6} \mathrm{M}_{\odot} \mathrm{yr}^{-1}$, consistent with the wide distribution of values inferred ${ }^{15,16,46}$ for nearby GRBs. However, the largescale ( $\gtrsim 1 \mathrm{kpc}$ ) environmental properties differ $^{40}$ from those of nearby GRB host galaxies, 
showing evidence for a super-solar metallicity that exceeds the proposed ${ }^{25,26}$ cut-off for relativistic explosions. We conclude that there is a broad diversity in the environments of engine-driven explosions, and therefore host galaxy properties alone cannot be used to discriminate between ordinary and engine-driven SNe.

With the advent ${ }^{47,48}$ of wide-field optical surveys (e.g., Palomar Transient Factory, PanSTARRS) the discovery rate of young, local SNe Ibc will effectively quadruple over the next 3-5 years (see Suppl. Info). Coupled with the ten-fold increase in the sensitivity of the Expanded Very Large Array (expected 2010; Ref. 51), relativistic SNe will be uncovered at an increased rate of $\sim 1$ per year within $d \lesssim 200$ Mpc. This is $\sim 3$ times higher than the rate at which nearby GRBs are discovered with current gamma-ray satellites. Thus, while such explosions have historically been found through their gamma-ray emission, long-wavelength surveys will soon provide a more powerful tool to pinpoint the nearest engine-driven supernovae.

Received 11 September 2018; Accepted draft.

1. Woosley, S. E. \& Bloom, J. S. The Supernova Gamma-Ray Burst Connection. Ann. Rev. Astr. Ap. 44, 507-556 (2006).

2. MacFadyen, A. I., Woosley, S. E. \& Heger, A. Supernovae, Jets, and Collapsars. Astrophys. J. 550, 410-425 (2001).

3. Piran, T. Gamma-ray bursts and the fireball model. Phys. Reports 314, 575-667 (1999).

4. Perna, R. \& Loeb, A. Constraining the Beaming of Gamma-Ray Bursts with Radio Surveys. Astrophys. J. 509, L85-L88 (1998).

5. Berger, E., Kulkarni, S. R., Frail, D. A. \& Soderberg, A. M. A Radio Survey of Type Ib and Ic Supernovae: Searching for Engine-driven Supernovae. Astrophys. J. 599, 408-418 (2003).

6. Soderberg, A. M., Nakar, E., Berger, E. \& Kulkarni, S. R. Late-Time Radio Observations of 68 Type Ibc Supernovae: Strong Constraints on Off-Axis Gamma-Ray Bursts. Astrophys. J. 638, 930-937 (2006).

7. Soderberg, A. M., Kulkarni, S. R., Nakar, E., Berger, E., Cameron, P. B. et al. Relativistic ejecta from X-ray flash XRF 060218 and the rate of cosmic explosions. Nature 442, 1014-1017 (2006). 
$6 \quad$ Soderberg et al.

8. Paragi, Z. TBD. Nature (2009).

9. Pignata, G., Maza, J., Antezana, R., Cartier, R., Folatelli, G. et al. in American Institute of Physics Conference Series (eds Giobbi, G., Tornambe, A., Raimondo, G., Limongi, M., Antonelli, L. A. et al.) 551-554 (2009).

10. Pignata, G., Maza, J., Hamuy, M., Antezana, R., Gonzalez, L. et al. Supernova 2009bb in NGC 3278. Central Bureau Electronic Telegrams 1731, 1-+ (2009).

11. Stritzinger, M., Philips, M. M., Morrell, N., Salgado, F. \& Folatelli, G. Supernova 2009bb in NGC 3278. Central Bureau Electronic Telegrams 1751, 1-+ (2009).

12. Soderberg, A. M. The Many Facets of Cosmic Explosions. Caltech PhD Thesis (2007).

13. Kulkarni, S. R., Frail, D. A., Wieringa, M. H., Ekers, R. D., Sadler, E. M. et al. Radio emission from the unusual supernova 1998bw and its association with the gamma-ray burst of 25 April 1998. Nature 395, 663-669 (1998).

14. Chevalier, R. A. Synchrotron Self-Absorption in Radio Supernovae. Astrophys. J. 499, 810-+ (1998).

15. Li, Z. \& Chevalier, R. A. Radio Supernova SN 1998BW and Its Relation to GRB 980425. Astrophys. J. 526, 716-726 (1999).

16. Soderberg, A. M., Kulkarni, S. R., Berger, E., Fox, D. W., Sako, M. et al. The sub-energetic $\gamma$-ray burst GRB 031203 as a cosmic analogue to the nearby GRB 980425. Nature 430, 648-650 (2004).

17. Chevalier, R. A. \& Fransson, C. Circumstellar Emission from Type Ib and Ic Supernovae. Astrophys. J. 651, 381-391 (2006).

18. Readhead, A. C. S. Equipartition brightness temperature and the inverse Compton catastrophe. Astrophys. J. 426, 51-59 (1994).

19. Tan, J. C., Matzner, C. D. \& McKee, C. F. Trans-Relativistic Blast Waves in Supernovae as Gamma-Ray Burst Progenitors. Astrophys. J. 551, 946-972 (2001).

20. Hurley, K., Cline, T., Mitrofanov, I. G., Golovin, D., Litvak, M. L. et al. in American Institute of Physics Conference Series (ed C. Meegan, C. Kouveliotou, \& N. Gehrels) 55-57 (2009). 
21. Pian, E., Amati, L., Antonelli, L. A., Butler, R. C., Costa, E. et al. BEPPOSAX Observations of GRB 980425: Detection of the Prompt Event and Monitoring of the Error Box. Astrophys. J. 536, 778-787 (2000).

22. Cappellaro, E., Evans, R. \& Turatto, M. A new determination of supernova rates and a comparison with indicators for galactic star formation. Astr. Astrophys. 351, 459-466 (1999).

23. Dahlen, T., Strolger, L.-G., Riess, A. G., Mobasher, B., Chary, R.-R. et al. High-Redshift Supernova Rates. Astrophys. J. 613, 189-199 (2004).

24. Levesque, E., Soderberg, A. M., Foley, R., Chakrabarty, S. \& others The High Metallicity Explosion Environment of SN 2009bb. ArXiv e-prints 0908.2818 (2009).

25. Woosley, S. E. \& Heger, A. The Progenitor Stars of Gamma-Ray Bursts. Astrophys. J. 637, 914-921 (2006).

26. Modjaz, M., Kewley, L., Kirshner, R. P., Stanek, K. Z., Challis, P. et al. Measured Metallicities at the Sites of Nearby Broad-Lined Type ic Supernovae and Implications for the Supernovae Gamma-Ray Burst Connection. Astron. J. 135, 1136-1150 (2008).

27. Law, N. M., Kulkarni, S. R., Dekany, R. G., Ofek, E. O., Quimby, R. M. et al. The Palomar Transient Factory: System Overview, Performance and First Results. ArXiv e-prints 0906.5350 (2009).

28. Kaiser, N., Aussel, H., Burke, B. E., Boesgaard, H., Chambers, K. et al. in Society of PhotoOptical Instrumentation Engineers (SPIE) Conference Series (eds Tyson, J. A. \& Wolff, S.) 154-164 (2002).

29. Perley, R. A., Napier, P. J. \& Butler, B. J. in Society of Photo-Optical Instrumentation Engineers (SPIE) Conference Series (ed Oschmann, Jr., J. M.) 784-795 (2004).

30. Blandford, R. D. \& McKee, C. F. Fluid dynamics of relativistic blast waves. Physics of Fluids 19, 1130-1138 (1976).

Supplementary Information is linked to the online version of the paper at www.nature.com/nature.

\section{Acknowledgements}

The VLA is operated by the National Radio Astronomy Observatory, a facility of the 
$8 \quad$ Soderberg et al.

National Science Foundation operated under cooperative agreement by Associated Universities, Inc. GMRT is run by the National Centre for Radio Astrophysics of the Tata Institute of Fundamental Research. This research has made use of the NASA/IPAC Extragalactic Database (NED) which is operated by the Jet Propulsion Laboratory, California Institute of Technology, under contract with the National Aeronautics and Space Administration. A.M.S. and O.F. acknowledge support by NASA through Hubble and GSRP grants, respectively. E.M.L. is funded through a Ford Foundation Predoctoral Fellowship. R.J.F. is a Clay fellow. R.A.C. and R.P.K. acknowledge support through NASA and NSF grants. G.P. and G.P. and M.H. acknowledge support from FONDECYT, Iniciativa Cientifica Milenio, FONDAP, and CONICYT. A.R. and S.C. are funded by an 11th Five Year Plan Project.

All authors contributed extensively to the work presented in this paper. Reprints and permissions information is available at npg.nature.com/reprintsandpermissions. Correspondence should be addressed to A. M. Soderberg (e-mail: asoderberg@cfa.harvard.edu). 
Figure 1: Radio observations of the nearest massive star explosions. The 8.46 $\mathrm{GHz}$ radio emission from $\mathrm{SN} 2009 \mathrm{bb}$ (red) is more luminous than any of the other 142 local $(d \lesssim 200 \mathrm{Mpc})$ SNe Ibc observed (Ref.12 and references within) to date on a comparable timescale $(\Delta t \lesssim 100$ days $)$, and is consistent ${ }^{46,13,16}$ with the radio afterglow luminosities of the nearest GRBs discovered through their gamma-ray signal within a similar volume (black). Local SNe Ibc with well-studied radio emission (grey) exhibit lower luminosities and peak at later times, indicating smaller sizes and lower mean expansion velocities. The radio emission from most local SNe Ibc is below our current detection threshold; we include them here as upper limits ( $3 \sigma$; grey triangles).

Figure 2: Synchrotron self-absorption model fits to the SN 2009bb radio spectra. The radio emission from GRBs and SNe Ibc is suppressed ${ }^{35,37}$ at low frequencies by SSA, defining the spectral peak frequency. The spectral shape below and above the peak is characterized as $F_{\nu} \propto \nu^{5 / 2}$ and $F_{\nu} \propto \nu^{-(p-1) / 2}$, respectively, where $p$ is the power-law index of the relativistic electron energy distribution above a minimum Lorentz factor, $\gamma_{m}$. Our multi-frequency radio observations of SN 2009bb taken with the VLA and GMRT (see Suppl. Info.) on Apr 8, May 10, Jun 6-10, and Aug 8-11 UT ( $\Delta t \approx 20,52,81$, and 145 days) are well described by a standard SSA spectrum, with $\nu_{p} \approx 6,3,1$, and $0.8 \mathrm{GHz}$, and peak flux densities of $F_{\nu, p} \approx 19,15,13$, and $11 \mathrm{mJy}$, respectively. As the blastwave expands the shocked material becomes optically thin, causing $\nu_{p}$ to cascade ${ }^{37}$ to lower frequencies with time. The optically thin spectral index is constrained to roughly $F_{\nu} \approx \nu^{-1}$ which implies $p \approx 3$, in line ${ }^{37}$ with other radio SNe Ibc. Error bars are $1 \sigma$.

Figure 3: Radio properties of the nearest massive star explosions directly reveal the blastwave velocities. We compare the peak radio luminosities for SNe Ibc (red) and nearby GRBs $(z \lesssim 0.1$; blue squares) as observed at the spectral peak frequency, $\nu_{p}$, and at time $\Delta t$. These observed properties are tightly related ${ }^{35}$ to the blastwave radius. The average velocities are reasonably estimated as $R / \Delta t$ (dashed grey lines). For SNe Ibc we infer typical velocities of $R / \Delta t \approx 0.1 \mathrm{c}$, while SN 2009bb (star) and the nearest GRBs show $R / \Delta t \approx c$. Error bars are $1 \sigma$. 
Figure 4: Blastwave velocity and energy for massive star explosions. We compare the blastwave velocities and inferred energies for the well-studied radio SNe Ibc and nearby GRBs included in Figure 3 with classic GRBs. Assuming equipartition of energy between electrons $\left(\epsilon_{e}\right)$ and magnetic fields $\left(\epsilon_{B}\right)$ as $\epsilon_{e}=\epsilon_{B}=0.33$ which accounts for equal energy in shocked protons, the total internal energy of the radio emitting source is $E \equiv E_{\min } / \epsilon_{B}$ where the minimum energy, $E_{\min }$, is derived ${ }^{37}$ from the energy density in magnetic fields, $E_{\min } \approx 8.3 \times 10^{46}(\mathrm{f} / 0.5)(B / \mathrm{G})^{2}\left(R / 10^{16} \mathrm{~cm}\right)^{3} \mathrm{erg}$, here $B \approx 0.43\left(\epsilon_{e} / \epsilon_{B}\right)^{-4 / 19}(f / 0.5)^{-4 / 19}\left(L_{\nu, p} / 10^{28} \mathrm{erg} \mathrm{s}^{-1} \mathrm{~Hz}^{-1}\right)^{-2 / 19}\left(\nu_{p} / 5 \mathrm{GHz}\right) \mathrm{G}$ and $f=0.5$ is the fraction of the spherical volume occupied by the radio-emitting region. For radio SNe Ibc (red; Ref. 12 and references therein), the average velocities are reasonably estimated as $\beta=R / c \Delta t$ since the bulk flow is ${ }^{37}$ expanding freely. The radio properties of these objects imply typical values of $\beta \approx 0.1$ and $E \approx 10^{47} \mathrm{erg}$. Adopting the same framework for SN $2009 \mathrm{bb}$ (yellow star) we find $R / \Delta t \approx 0.9 c$ and $E \approx 10^{49} \mathrm{erg}$ and note that the inclusion of relativistic effects (e.g., photon arrival time) would not change these results significantly as shown by Ref. 13 and 15 for GRB 980425/SN 1998bw. The nearest GRBs (blue squares) tend to be trans-relativistic and some decelerate rapidly, so we adopt the inferred ${ }^{46,13,15,16}$ blastwave velocities and energies from the earliest available radio data, at $\Delta t \lesssim 5$ days. Likewise, the inferred velocity of SN $2009 \mathrm{bb}$ at $\Delta t \approx 20$ days, is a strict lower limit since there is evidence that the blastwave decelerated early on (see Suppl. Info.). Finally, classic GRBs (blue circles) in the decelerating Blandford-McKee phase ${ }^{34}$ have $\Gamma \propto t^{-3 / 8}$ so we conservatively estimate their blastwave velocity at $\Delta t=1$ day according to $\Gamma \propto t^{-3 / 8}$ and adopt the beaming corrected blastwave energies from afterglow modeling (Ref. 6 and references therein). Error bars are $1 \sigma$. 


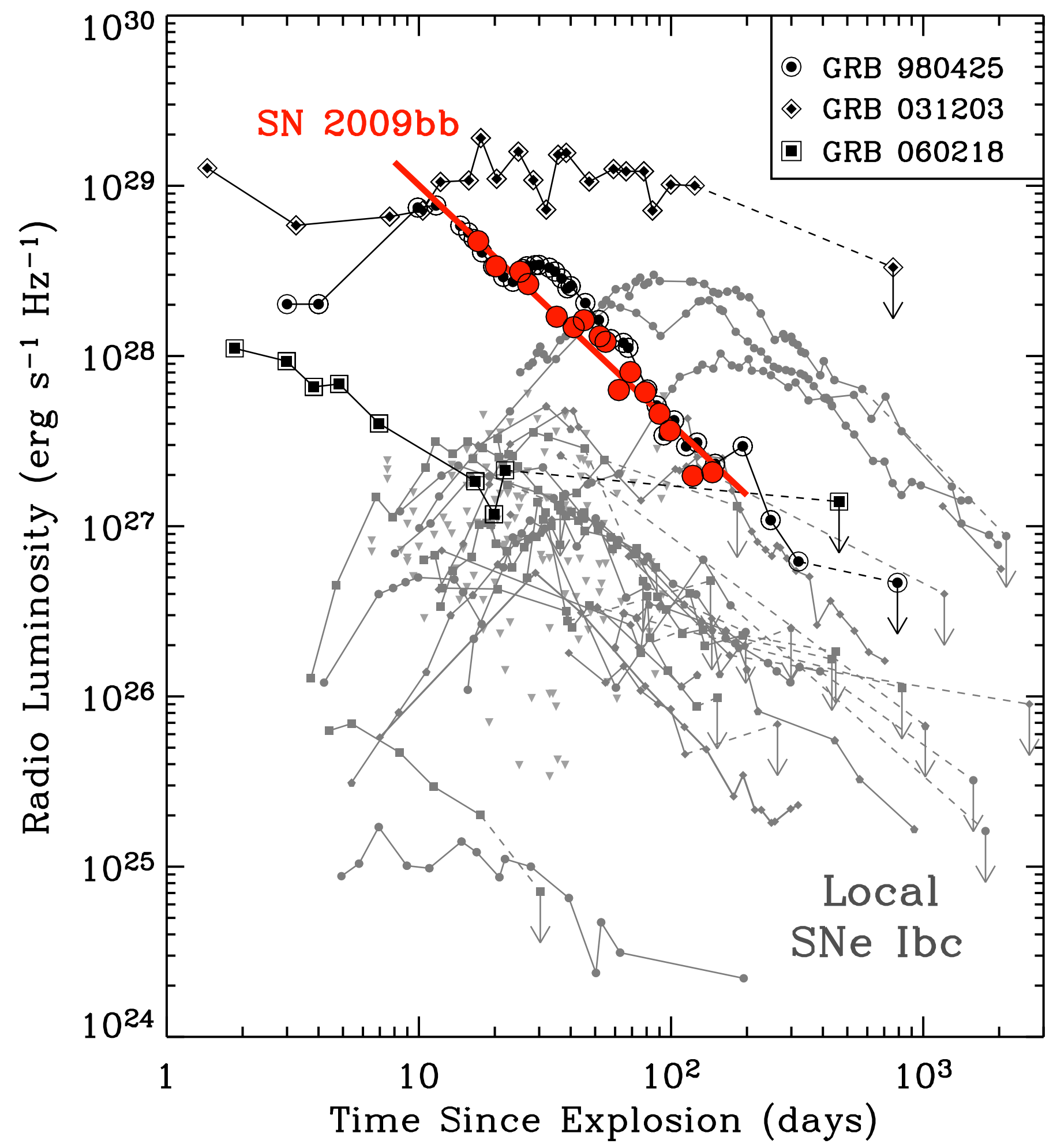

Figure 1. 


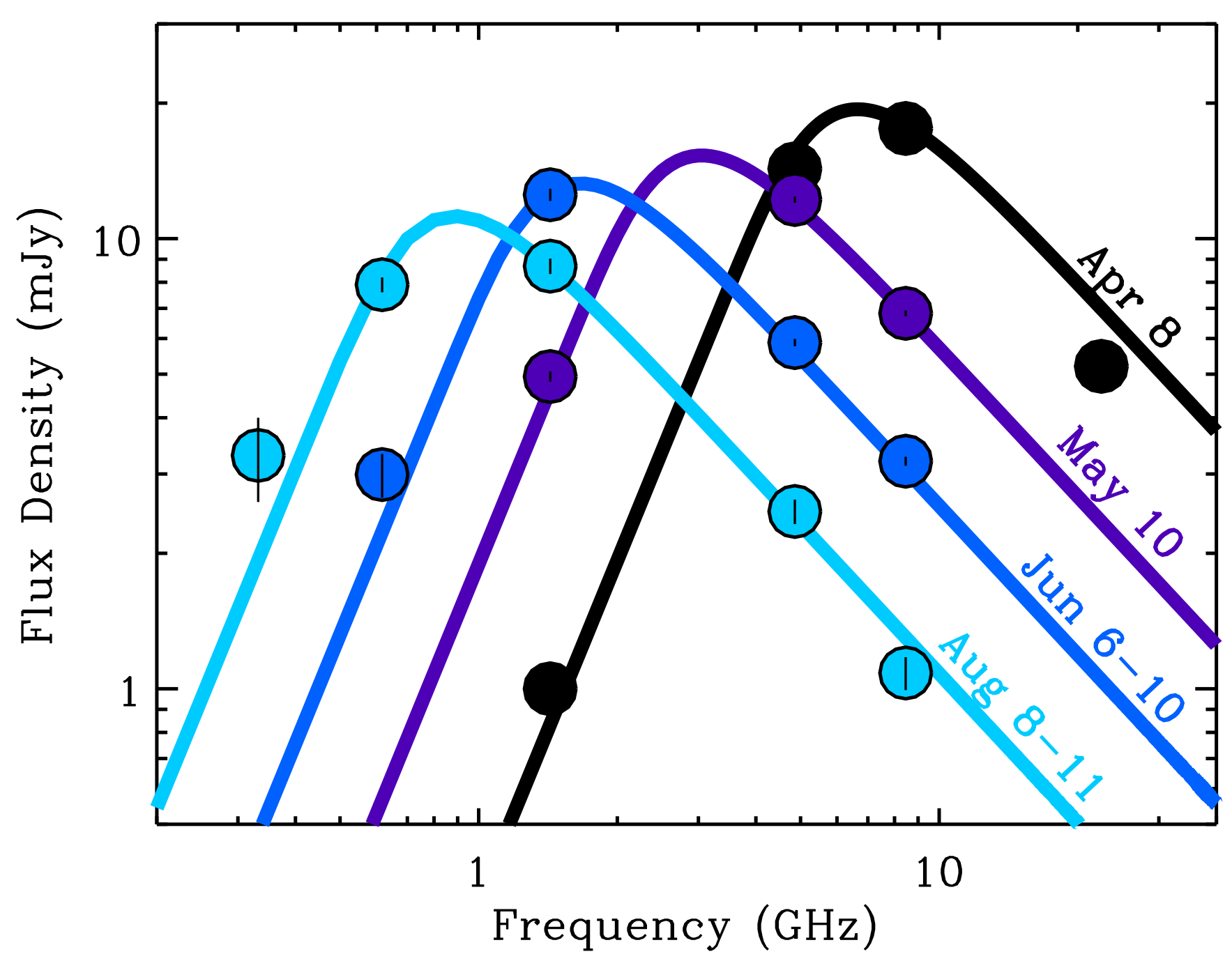

Figure 2. 


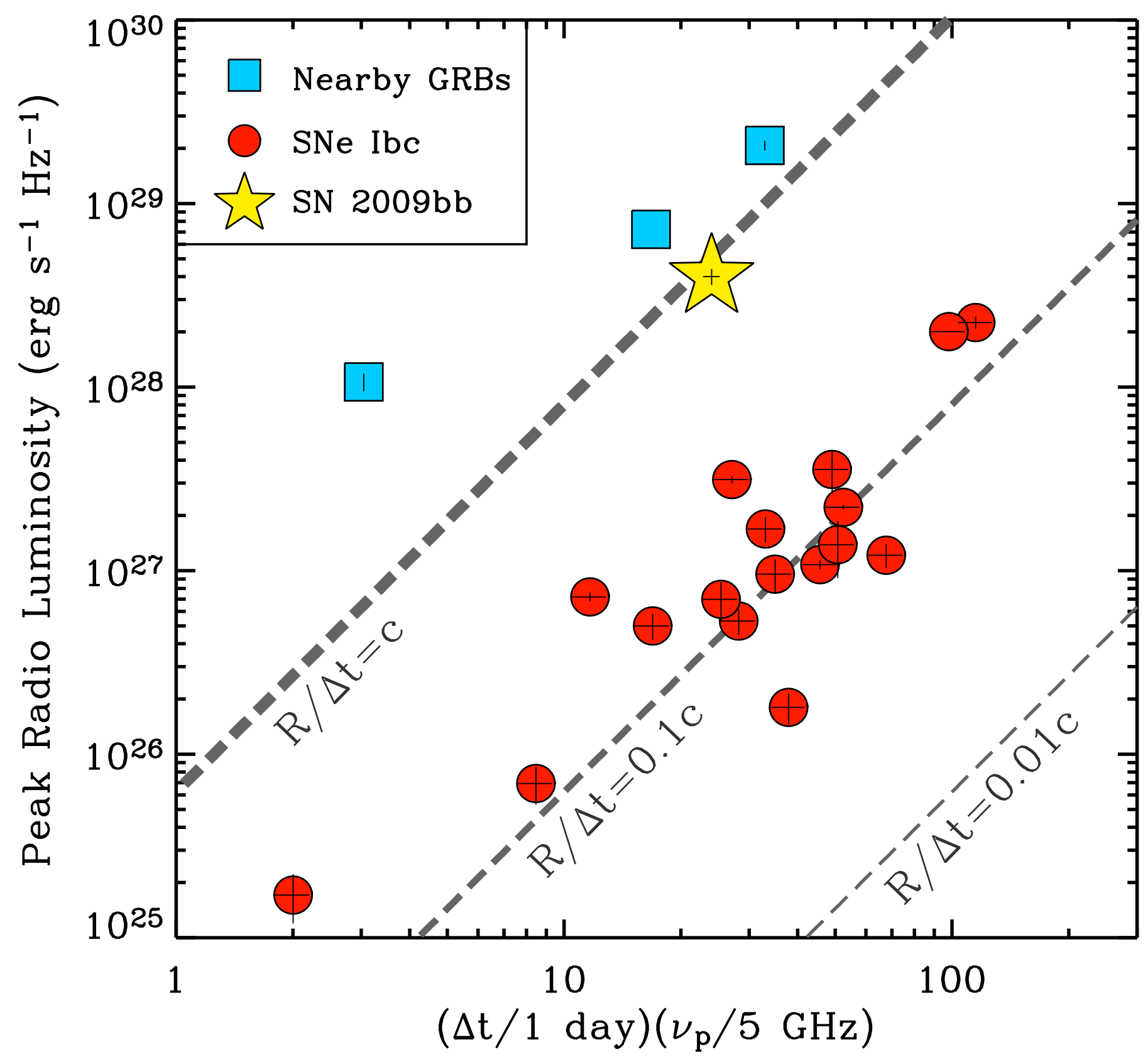

Figure 3. 


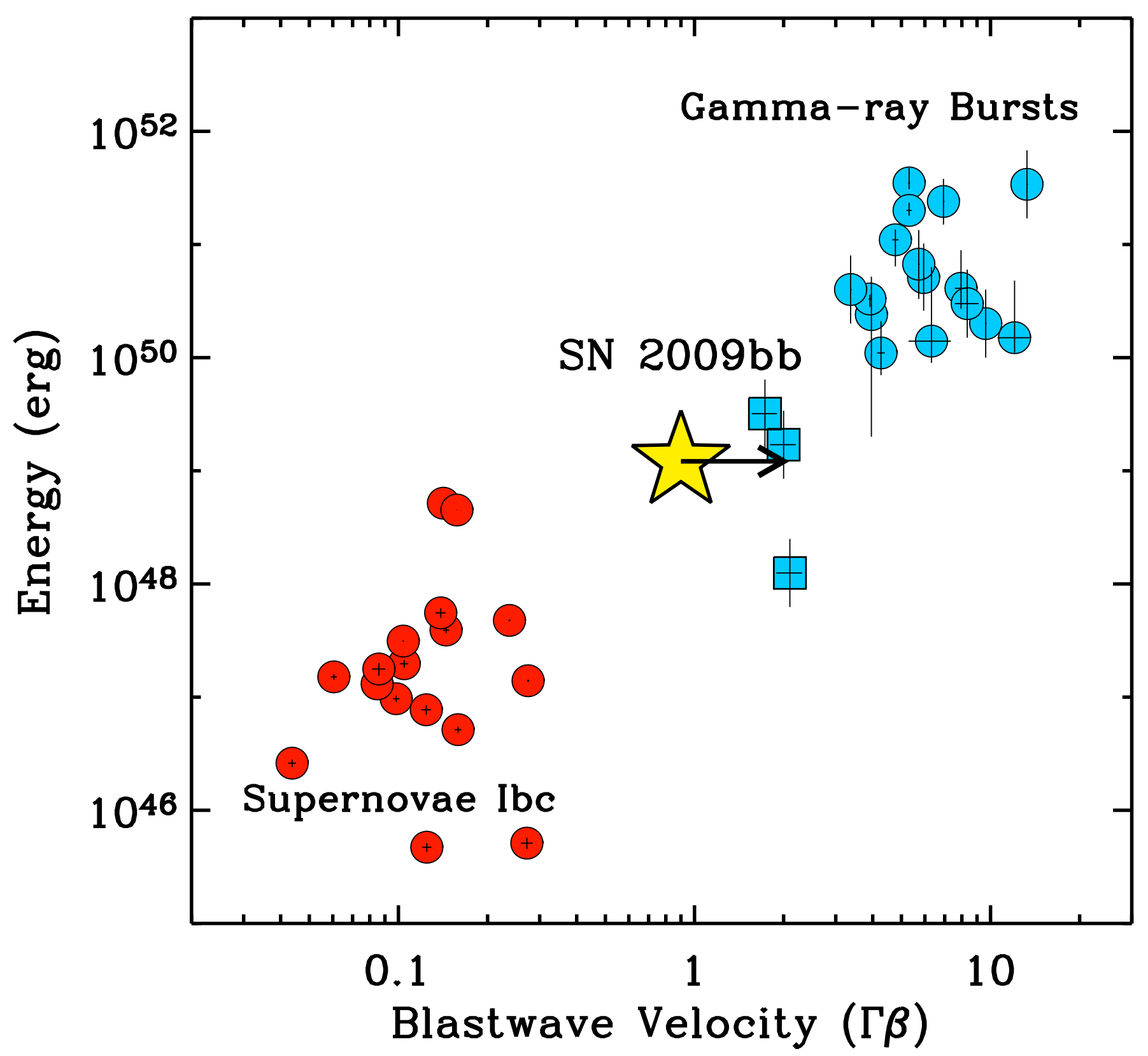

Figure 4 . 


\section{SUPPLEMENTARY INFORMATION}

\section{A Relativistic Supernova Powered by a Central Engine but Lacking a Detected $\gamma$-ray Burst}

\section{Explosion Date}

The Chilean Automatic Supernova Search Program (CHASE; Ref. 31) and the Carnegie Supernova Project (CSP; Ref. 32) initiated a dedicated optical and near-IR follow-up campaign for SN 2009bb beginning immediately after discovery. Using a combination of pre- and post-explosion data collected by CHASE and CSP a well-sampled optical lightcurve has been constructed (G. P., M. D. S. \& M. A. H. in prep). Fitting a expanding fireball model, $L_{\mathrm{opt}} \propto t^{2}$, to the rising component of the optical light-curve implies an explosion date of $t_{0} \approx$ Mar $18.4 \mathrm{UT} \pm 0.6$ days. While this fit enables a first estimate of the explosion date, it assumes a constant velocity. Realistically some deceleration is expected, especially for fast expanding SNe like SN 2009bb and SN 1998bw so it is reasonable that the explosion time should be somewhat later that the fireball fit. Along this line, we match the early evolution to that observed ${ }^{33}$ for SN 1998bw and find a slightly later explosion date, $t_{0} \approx$ Mar 20 UT. We adopt an intermediate value of $t_{0}=$ Mar $19 \pm 1$ UT throughout the paper. Finally, we note that the inferred early discovery is consistent with the observed epoch of maximum light, Apr 1 UT, occurring two weeks after our estimated explosion date and in line with the observed rise times for other SNe Ibc (G. P., M. D. S. \& M. A. H. in рrep).

\section{Radio Observations}

\subsection{Very Large Array observations}

We observed SN 2009bb with the Very Large Array (VLA) on many epochs spanning Apr 5.2 to Jun 6.0 UT. Data were collected at four frequencies, $\nu_{\mathrm{obs}}=1.43,4.86,8.46$ and 22.5 GHz. All VLA observations were obtained in the standard continuum mode with $2 \times 50 \mathrm{MHz}$ bands. At $22.5 \mathrm{GHz}$ we included referenced pointing scans to correct for the systematic 10-20 arcsec pointing errors of the VLA antennas. We used 3C286 (J1331+305) 
for flux calibration, while phase referencing was performed against calibrator J1036-3744. Data were reduced using standard packages within the Astronomical Image Processing System (AIPS). Flux density measurements were obtained by fitting a Gaussian model to the radio emission. For observations at $1.43 \mathrm{GHz}$ we restricted the UV-range to reduce contamination from diffuse host galaxy emission from the strongly star-formation region in which the SN resides (Figure 5). The resulting flux density measurements are listed in Table 1 and shown in Figure 6.

\subsection{Giant Meterwave Radio Telescope Observations}

We observed the SN 2009bb with the Giant Meterwave Radio Telescope (GMRT) on several epochs spanning May 31 and Aug 10 UT at effective frequencies of 1287, 617, and $332 \mathrm{MHz}$. We used a $2 \times 16 \mathrm{MHz}$ bandwidth each divided into 128 frequency channels. The duration of the observations was two hours each, except for the $332 \mathrm{MHz}$ observation which was $4 \mathrm{hrs}$ in duration. We used 3C147 and 3C286 for flux and bandpass calibration and J1057-245, J1107-448, and J1018-317 to monitor the phase at 1287, 617, and $332 \mathrm{MHz}$, respectively.

The GMRT data were reduced and analyzed with AIPS. To remove contamination from diffuse host galaxy emission, we removed the short baselines $0-5 \mathrm{k} \lambda$ and $0-3 \mathrm{k} \lambda$ at 1287 and $617 \mathrm{MHz}$, respectively. At $332 \mathrm{MHz}$ the host galaxy emission dominates that of the $\mathrm{SN}$ at the SN position. To estimate the flux density of the diffuse emission in the beam of the SN, we first construct a host galaxy map at $617 \mathrm{MHz}$ and measure an integrated flux density of $F_{617 \mathrm{MHz}}=113.5 \pm 5.7 \mathrm{mJy}$ after removal of the SN emission. We convolve this map down to the beam size of our $332 \mathrm{MHz}$ map, using the AIPS task CONVL. The extended emission at the $\mathrm{SN}$ site is then scaled to $332 \mathrm{MHz}$ using the spectral index $\left(F_{\nu} \propto \nu^{-0.87}\right)$ of the host galaxy emission as measured between 1287 and $617 \mathrm{MHz}$. The $332 \mathrm{MHz}$ map is then re-gridded to match the convolved $617 \mathrm{MHz}$ map using the AIPS task HGEOM. The SN flux at the $332 \mathrm{MHz}$ is then determined as the excess observed flux in the synthesized beam over and above the estimated extended emission at the SN position. Our resulting flux densities for all GMRT epochs are listed in Table 2 and we note that the error in the SN flux density at $332 \mathrm{MHz}$ is dominated by the uncertainty in determining the extended emission at $617 \mathrm{MHz}$. 


\section{Radio Temporal Evolution}

The temporal and spectral evolution of the synchrotron emission is determined by the dynamics, the density profile of the ejecta, and the properties of the circumstellar medium. Young, non-relativistic SNe expand freely since their ejecta are largely undecelerated while GRBs expand ${ }^{34}$ according to the Blandford-McKee solution for relativistic, decelerated blastwaves. Both SNe and GRBs ultimately enter a phase of non-relativistic adiabatic expansion, the Sedov phase, when the ejecta have fully decelerated and the transverse velocity falls well below $\Gamma \beta \lesssim 1$. Trans-relativistic blastwaves, for which $\Gamma \beta \sim$ a few, e.g., SN 1998bw/GRB 980425, bridge these three hydrodynamic regimes and the flux density evolution depends on the structure of the ejecta.

The early ( $\Delta t \lesssim 40$ days) radio emission of SN 2009bb decays at both optically-thick and -thin frequencies (Figure 6), in contrast to the predicted ${ }^{35}$ evolution for SNe Ibc in free expansion. This indicates that another process is responsible for powering the radio emission at early time: a central engine. In this context, the early evolution may be understood if the geometry of the relativistic outflow was originally aspherical. In this scenario, the relativistic ejecta are detached from the freely expanding SN outflow and decelerate $^{36}$ on a timescale, $t_{\text {dec }} \approx 1\left(E / 10^{49} \mathrm{erg}\right)\left(\dot{M} / 10^{-5} \mathrm{M}_{\odot} \mathrm{yr}^{-1}\right)^{-1}$ day, when $\Gamma \beta \lesssim 1$. Here, $\dot{M}=4 \pi \rho v_{w} r^{2}$ is the mass loss rate of the progenitor star, $\rho$ is the circumstellar density and $v_{w}$ is the velocity of the progenitor wind. Based on the SSA model fits to the radio spectra, we estimate ${ }^{37}$ the mass loss rate of the $\mathrm{SN} 2009 \mathrm{bb}$ progenitor to be $\dot{M} \approx 6.4 \times$ $10^{-7}\left(\epsilon_{e} / \epsilon_{B}\right)^{-8 / 19}\left(\epsilon_{B} / 0.33\right)^{-1}(f / 0.5)^{-8 / 19}\left(F_{\nu, p} / \mathrm{mJy}\right)^{-4 / 19}\left(\nu_{p} / 5 \mathrm{GHz}\right)^{2}(\Delta t / 10 \text { days })^{2} \mathrm{M}_{\odot} \mathrm{yr}^{-1}$ or $\dot{M} \approx 2.0 \times 10^{-6} \mathrm{M}_{\odot} \mathrm{yr}^{-1}$ for $f=0.5$ and $\epsilon_{e}=\epsilon_{B}=0.33$ at $\Delta t \approx 20$ days. Here we have assumed a stellar wind density profile, $\rho \propto r^{-2}$, and a steady progenitor wind velocity of $v_{w} \approx 10^{3} \mathrm{~km} \mathrm{~s}^{-1}$ consistent with the observed ${ }^{38}$ properties for local Wolf-Rayet stars. For $\mathrm{SN} 2009 \mathrm{bb}$, we estimate that the outflow decelerates by $t_{\mathrm{dec}} \approx 6$ days and note that this estimate is independent of the partition fractions. Therefore the mean expansion velocity of $R / \Delta t \approx 0.9 c$ inferred at $\Delta t \approx 20$ days is a strict lower limit on the initial velocity of the outflow. Thereafter the ejecta spread ${ }^{39}$ sideways, stalling the radial expansion as the outflow becomes spherical and causing the peak radio emission to decay. The blastwave spherizes ${ }^{36}$ on a timescale, $t_{\mathrm{sph}} \approx 6\left(E / 10^{49} \mathrm{erg}\right)\left(\dot{M} / 10^{-5} \mathrm{M}_{\odot} \mathrm{yr}^{-1}\right)^{-1}$ days. By $\Delta t \approx 34$ 
days, we estimate that the SN 2009bb blastwave was spherical and the radio evolution thereafter is well described by a standard spherically symmetric model.

\section{X-ray Observations}

\subsection{Chandra data}

We observed SN 2009bb with Chandra ACIS-S beginning at Apr $18.6 \mathrm{UT}$ ( $\Delta t \approx 31$ days) for $10 \mathrm{ksec}$ under our approved Cycle 10 program to study the X-ray properties of radioemitting Type Ibc supernovae. We detect an X-ray source coincident with the optical and radio counterparts at position $\alpha(\mathrm{J} 2000)=10^{\mathrm{h}} 31^{\mathrm{m}} 33.86^{\mathrm{s}}$ and $\delta(\mathrm{J} 2000)=-39^{\circ} 57^{\prime} 29.4^{\prime \prime}( \pm 0.4$ arcsec in each coordinate). The data were reduced and the source counts were extracted in the standard manner using the Chandra threads. Within a 4.9 arcsec aperture we extract 24 counts. After correcting for an inferred foreground extinction of $E(B-V) \approx 0.5$ (see Ref. 40) corresponding ${ }^{41}$ to a column density, $n_{H}=2.92 \times 10^{21} \mathrm{~cm}^{-2}$, we estimate an (absorbed) unabsorbed flux of $\left(F_{X}=1.5 \pm 0.3 \times 10^{14} \mathrm{erg} \mathrm{cm}^{-2} \mathrm{~s}^{-1}\right) F_{X}=2.3 \pm 0.5 \times$ $10^{-14} \mathrm{erg} \mathrm{cm}^{-2} \mathrm{~s}^{-1}(0.3-10 \mathrm{keV})$ assuming a power-law spectrum with photon index, $\Gamma=2$.

At the distance of NGC 3278, the unabsorbed flux corresponds to an X-ray luminosity of $L_{X}=4.4 \pm 0.9 \times 10^{39} \mathrm{erg} \mathrm{s}^{-1}$. The X-ray emission from SN 2009bb is therefore a factor of a few less luminous than GRB 980425 observed on a comparable timescale and at the high end of the X-ray luminosities observed ${ }^{37}$ for other local SNe Ibc.

\subsection{Swift X-ray Telescope Data}

We also observed SN 2009bb with the Swift X-ray Telescope (XRT) on several epochs spanning Mar 24.1 to Apr 18.7 UT. Data were retrieved from the HEASARC archive and reduction performed with the xrtpipeline script packaged within the HEAsoft software. We used the default grade selections and screening parameters to extract measurements for the $0.3-10 \mathrm{keV}$ energy range.

We extracted the counts within a $47 \operatorname{arcsec}(90 \%$ PSF containment) aperture centered on the optical SN position in each epoch. Diffuse emission from the host galaxy is clearly detected within the extraction region. We bootstrap the flux calibration to the Chandra data, temporally coincident with our final XRT epoch. Adopting the same 47 arcsec 
aperture for the Chandra data, we extract 233 counts after subtracting off the background count rate. Fitting the same absorbed power-law model described above, we find an unabsorbed flux of $F_{X}=3.0 \pm 0.8 \times 10^{-13} \mathrm{erg} \mathrm{cm}^{-2} \mathrm{~s}^{-1}$. Therefore, within the XRT PSF, the host galaxy emission dominates that of the SN by a factor of $\sim 10$. Since the fractional uncertainties of the XRT fluxes preclude our ability to extract estimates of the SN flux, we adopt the measurements as upper limits (see Table 3). Finally, we note that a comparison of the first XRT upper limit and the measured SN flux from the Chandra observation constrain the temporal evolution of the SN X-ray flux to be no steeper than $F_{X} \propto t^{-1}$.

The flux ratio between radio and X-ray band can reveal the nature of the X-ray emission which, in the case of SNe Ibc, may be dominated by (i) thermal emission associated with the shock-heated ejecta, (ii) non-thermal synchrotron emission from the shocked circumstellar material, or (iii) inverse Compton up-scattering of optical photons by radio-emitting electrons (see Ref. 37 for a review). In the case of SN 2009bb, at the epoch of the Chandra observation we measure the spectral index between the radio $\left(\nu_{R}=8.46 \mathrm{GHz}\right)$ and X-ray band $\left(\nu_{X}=2 \times 10^{17} \mathrm{~Hz}\right)$, to be $F_{\nu} \propto \nu^{-0.82 \pm 0.02}$. This is somewhat flatter than that expected by an extrapolation of the radio synchrotron spectrum as $F_{\nu} \propto \nu^{-1}$. Given that the optical SN emission peaks on a similar timescale, it is feasible that the X-ray emission is dominated by inverse Compton emission. Alternatively, if the shock is cosmic ray dominated the synchrotron spectrum flattens ${ }^{37}$ at higher frequencies. The nature of the X-ray emission will be discussed in a subsequent paper.

\section{Searches for a Coincident High Energy Signal}

Massive star explosions can give rise to high energy emission at the moment of explosion via two primary channels: (i) strong X-ray and/or gamma-ray emission powered by ultrarelativistic ejecta as inferred for GRBs, and (ii) weak X-ray emission produced by shock breakout in ordinary non-relativistic supernova explosions (e.g., SN 2008D). In order to search for a statistically significant association between an optical SN and a high energy transient, an accurate estimate of the explosion time is required. SN 2009bb is unique among local optically-discovered SNe in that the optical data set is sufficient to enable a 
reasonably tight constraint on the explosion date $(\S 1)$. Along this line, we searched for high energy counterparts between the dates Mar 18-20 UT and coincident with the SN position.

Among the automatic triggered public bursts announcements made through the Gamma-Ray Burst Coordination Network (GCN) from Swift, Fermi, Integral, Agile, Suzaku and the Interplanetary Network (IPN), we note just one marginally coincident burst. The weak Fermi Gamma-ray Burst Monitor (GBM) burst GRB 090320C was detected on Mar $20.045 \mathrm{UT}$ at $\alpha(\mathrm{J} 2000)=07^{\mathrm{h}} 13^{\mathrm{m}}, \delta(\mathrm{J} 2000)=-43^{\circ} 18^{\prime}(1 \sigma$ positional uncertainty of $15 \mathrm{deg}$; Ref. 42 ) and lies $3.1 \sigma$ away from the optical and radio SN 2009bb positions. With a duration of $T_{90} \approx 4 \mathrm{sec}$, the flux of the burst is $F_{\gamma}=3.9 \times 10^{-7} \mathrm{erg} \mathrm{cm}^{-2} \mathrm{~s}^{-1}$ (8$1000 \mathrm{keV}$ ). Given the large positional uncertainty due to the weakness of the burst, we undertook a Monte Carlo experiment to calculate the probability that GRB 090320C was associated with SN 2009bb. We used 1000 iterations to test the frequency that a GRB with the properties observed for GRB 090320C could be recovered from a burst within $1 \sigma$ of the location of SN 2009bb given the known detector response. From this experiment, we exclude the association of GRB 090320C and SN 2009bb at the $99 \%$ confidence level. Separately, we note that the SN position was in the field-of-view of the GBM for 62 percent of our bracketed explosion date estimate.

We next searched for sub-threshold bursts in the Swift Burst Alert Telescope (BAT) data, including those data from the BAT Slew Survey (BATSS; Ref. 43) for the same range of dates. The SN position was within the 50 deg field-of-view of the BAT for just 7 percent of this time. With an accuracy of $\sim 2$ arcmin, no sub-threshold $\left(F_{\gamma} \gtrsim 5.5 \sigma\right.$ above background) triggers were detected at a position coincident with SN 2009bb. Moreover, in the BATSS data set we find no statistically significant $\left(F_{\gamma} \gtrsim 3.5 \sigma\right)$ bursts during the spacecraft slews over this timescale. We also note that GBM burst 090320C was not in the field-of-view of the BAT at the time of the Fermi trigger.

By combining the monitoring times of BAT and GBM and accounting for the 9772 seconds when the SN position was monitored by both satellites simultaneously, we estimate a combined monitoring time of 65 percent. Furthermore, both GBM and BAT are included within the all-sky Interplanetary Network (IPN; ) of gamma-ray satellites which offers 100\% 
temporal coverage to SN 2009bb over the bracketed explosion date estimate. Based on the detection efficiency of IPN as reported in Ref. 44, we place an upper limit on the detectable gamma-ray fluence from SN 2009bb of $F_{\gamma} \lesssim 5 \times 10^{-6} \mathrm{erg} \mathrm{cm}^{-2}$ (band, $25-150 \mathrm{keV}$ ). At a distance of $d \approx 40 \mathrm{Mpc}$, this corresponds to an isotropic-equivalent energy release of $E_{\gamma} \lesssim 10^{48}$ erg which is a factor of two higher than that observed ${ }^{45}$ from GRB 980425.

The lack of a detected gamma-ray counterpart can be attributed to several factors. For example, if the GRB jet is not directed towards our line-of-sight, relativistic beaming inhibits the detection of gamma-rays. Most importantly, as seen in the case of the nearest GRBs, the gamma-rays can be exceedingly weak and the isotropic gamma-ray energy yield dwarfed by that of the blastwave kinetic energy (e.g., GRB 980425; Ref. 45). Along this line we note that the rate of such intrinsically weak GRBs at $z \lesssim 0.1$ is $3-10$ times higher ${ }^{46}$ than that of classic GRBs discovered preferentially at larger redshifts.

\section{Predictions for the Rate of Engine-driven SNe from Long-Wavelength Sur- veys}

Local $(d \lesssim 200 \mathrm{Mpc})$ SNe Ibc are currently discovered* at a rate of $\sim 25 \mathrm{yr}^{-1}$ by the ongoing efforts of amateur astronomers and automated searches (e.g., Lick Observatory Supernova Search, CHASE) that monitor weekly a sample of nearby galaxies searching for new or transient objects. With the advent of wide-field optical surveys, specifically The Palomar Transient Factory (PTF; Ref. 47) and Pan-STARRS, ${ }^{48}$ this sample will be supplemented by similarly nearby and young SNe Ibc at a rate of $\sim 50 \mathrm{yr}^{-1}$ (PTF) and $\sim 25 \mathrm{yr}^{-1}$ (Pan-STARRS) over the next 3-5 yrs given the volumetric rate ${ }^{49,50}$ of SNe Ibc of $1.7 \times 10^{4} \mathrm{Gpc}^{-3} \mathrm{yr}^{-1}$.

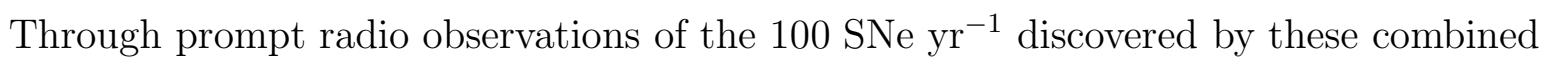
efforts, engine-driven events like SN 2009bb can be clearly identified by their luminous and low-frequency radio spectra. The current sensitivity of the Very Large Array $(3 \sigma \approx 0.1$ mJy in $15 \mathrm{~min}$ ) implies that the radio luminosity light-curve of SN 2009bb extending to $\sim 100$ days could be detected with $\mathrm{S} / \mathrm{N} \gtrsim 5$ out to a distance of $130 \mathrm{Mpc}$. The increase in sensitivity of the Expanded-VLA (expected 2010; Ref. 51) will enable such events to *http://www.cfa.harvard.edu/iau/lists/Supernovae.html 
be detected significantly further, to $400 \mathrm{Mpc}$, well matched to the volumes probed by the optical surveys, and requiring just $25 \mathrm{hrs}$ of observing time per year. Given our estimated fraction of engine-driven events, $0.7_{-0.6}^{+1.6 \%}$, we anticipate that $\sim 1$ new relativistic SN will be both discovered and identified each year. This is a factor of three higher than the current discovery rate ${ }^{46}$ of nearby GRBs with gamma-ray satellites, demonstrating that long-wavelength surveys will ultimately become powerful tools in the discovery of such events. In fact, over the next decade, the "deep-wide-fast" optical survey planned for the Large Synoptic Survey Telescope (LSST; Ref. 52) will reveal $\sim 500$ SNe Ibc each year within $400 \mathrm{Mpc}$, of which up to $\sim 10$ will be engine-driven.

Received 11 September 2018; Accepted draft.

31. Pignata, G., Maza, J., Antezana, R., Cartier, R., Folatelli, G. et al. in American Institute of Physics Conference Series (eds Giobbi, G., Tornambe, A., Raimondo, G., Limongi, M., Antonelli, L. A. et al.) 551-554 (2009).

32. Hamuy, M., Folatelli, G., Morrell, N. I., Phillips, M. M., Suntzeff, N. B. et al. The Carnegie Supernova Project: The Low-Redshift Survey. Publ. Astr. Soc. Pacific 118, 2-20 (2006).

33. Galama, T. J., Vreeswijk, P. M., van Paradijs, J., Kouveliotou, C., Augusteijn, T. et al. An unusual supernova in the error box of the gamma-ray burst of 25 April 1998. Nature 395, 670-672 (1998).

34. Blandford, R. D. \& McKee, C. F. Fluid dynamics of relativistic blast waves. Physics of Fluids 19, 1130-1138 (1976).

35. Chevalier, R. A. Synchrotron Self-Absorption in Radio Supernovae. Astrophys. J. 499, 810-+ (1998).

36. Waxman, E. The Nature of GRB 980425 and the Search for Off-Axis Gamma-Ray Burst Signatures in Nearby Type Ib/c Supernova Emission. Astrophys. J. 602, 886-891 (2004).

37. Chevalier, R. A. \& Fransson, C. Circumstellar Emission from Type Ib and Ic Supernovae. Astrophys. J. 651, 381-391 (2006).

38. Cappa, C., Goss, W. M. \& van der Hucht, K. A. A Very Large Array 3.6 Centimeter Continuum Survey of Galactic Wolf-Rayet Stars. Astron. J. 127, 2885-2897 (2004). 
39. Sari, R., Piran, T. \& Halpern, J. P. Jets in Gamma-Ray Bursts. Astron. J. 519, L17-L20 (1999).

40. Levesque, E., Soderberg, A. M., Foley, R., Chakrabarty, S. \& others The High Metallicity Explosion Environment of SN 2009bb. ArXiv e-prints 0908.2818 (2009).

41. Predehl, P. \& Schmitt, J. H. M. M. X-raying the interstellar medium: ROSAT observations of dust scattering halos. A\&A 293, 889-905 (1995).

42. Chaplin, V. GRB 090320C: Fermi GBM Detection. GRB Circular Network 9022, 1-+ (2009).

43. Grindlay, J. E., Copete, A., Allen, B., Hong, J., Barthelmy, S. et al. in AAS/High Energy Astrophysics Division 19.05-+ (2008).

44. Hurley, K., Cline, T., Mitrofanov, I. G., Golovin, D., Litvak, M. L. et al. in American Institute of Physics Conference Series (ed C. Meegan, C. Kouveliotou, \& N. Gehrels) 55-57 (2009).

45. Pian, E., Amati, L., Antonelli, L. A., Butler, R. C., Costa, E. et al. BEPPOSAX Observations of GRB 980425: Detection of the Prompt Event and Monitoring of the Error Box. Astrophys. J. 536, 778-787 (2000).

46. Soderberg, A. M., Kulkarni, S. R., Nakar, E., Berger, E., Cameron, P. B. et al. Relativistic ejecta from X-ray flash XRF 060218 and the rate of cosmic explosions. Nature 442, 1014-1017 (2006).

47. Law, N. M., Kulkarni, S. R., Dekany, R. G., Ofek, E. O., Quimby, R. M. et al. The Palomar Transient Factory: System Overview, Performance and First Results. ArXiv e-prints 0906.5350 (2009).

48. Kaiser, N., Aussel, H., Burke, B. E., Boesgaard, H., Chambers, K. et al. in Society of PhotoOptical Instrumentation Engineers (SPIE) Conference Series (eds Tyson, J. A. \& Wolff, S.) 154-164 (2002).

49. Cappellaro, E., Evans, R. \& Turatto, M. A new determination of supernova rates and a comparison with indicators for galactic star formation. Astr. Astrophys. 351, 459-466 (1999).

50. Dahlen, T., Strolger, L.-G., Riess, A. G., Mobasher, B., Chary, R.-R. et al. High-Redshift Supernova Rates. Astrophys. J. 613, 189-199 (2004).

51. Perley, R. A., Napier, P. J. \& Butler, B. J. in Society of Photo-Optical Instrumentation Engineers (SPIE) Conference Series (ed Oschmann, Jr., J. M.) 784-795 (2004). 
24 Soderberg et al.

52. Ivezic, Z., Tyson, J. A., Allsman, R., Andrew, J., Angel, R. et al. LSST: from Science Drivers to Reference Design and Anticipated Data Products. ArXiv e-prints 0805.2366, (2008). 


\begin{tabular}{|c|c|c|c|c|c|}
\hline $\begin{array}{l}\text { Date } \\
(\mathrm{UT})\end{array}$ & $\begin{array}{c}F_{\nu, 1.43 \mathrm{GHz}} \\
(\mathrm{mJy})\end{array}$ & $\begin{array}{c}F_{\nu, 4.86 \mathrm{GHz}} \\
(\mathrm{mJy})\end{array}$ & $\begin{array}{c}F_{\nu, 8.46 \mathrm{GHz}} \\
(\mathrm{mJy})\end{array}$ & $\begin{array}{c}F_{\nu, 22.5 \mathrm{GHz}} \\
(\mathrm{mJy})\end{array}$ & $\begin{array}{c}\text { VLA } \\
\text { Config. }\end{array}$ \\
\hline Apr 5.2 & - & - & $24.681 \pm 0.066$ & - & $\mathrm{B}$ \\
\hline Apr 8.2 & $1.308 \pm 0.099$ & $12.863 \pm 0.161$ & $17.568 \pm 0.088$ & $5.204 \pm 0.191$ & B \\
\hline Apr 13.2 & - & - & $16.349 \pm 0.107$ & - & B \\
\hline Apr 15.1 & - & $9.629 \pm 0.181$ & $13.812 \pm 0.114$ & $5.170 \pm 0.394$ & B \\
\hline Apr 23.2 & $1.141 \pm 0.159$ & $7.601 \pm 0.226$ & $8.881 \pm 0.121$ & - & B \\
\hline Apr 29.1 & $2.963 \pm 0.348$ & $7.632 \pm 0.202$ & $7.714 \pm 0.095$ & $1.796 \pm 0.240$ & B \\
\hline May 3.1 & $3.406 \pm 0.122$ & $9.680 \pm 0.165$ & $8.482 \pm 0.098$ & - & B \\
\hline May 10.1 & $4.939 \pm 0.132$ & $12.215 \pm 0.196$ & $6.824 \pm 0.102$ & - & B \\
\hline May 13.0 & $5.836 \pm 0.136$ & $9.727 \pm 0.508$ & $6.327 \pm 0.151$ & - & B \\
\hline May 20.1 & $6.903 \pm 0.180$ & $8.542 \pm 0.172$ & $3.294 \pm 0.118$ & - & B \\
\hline May 27.0 & $6.702 \pm 0.351$ & $8.568 \pm 0.080$ & $4.204 \pm 0.060$ & - & $\mathrm{CnB}$ \\
\hline Jun 6.0 & $9.465 \pm 0.186$ & $5.877 \pm 0.110$ & $3.203 \pm 0.074$ & - & $\mathrm{CnB}$ \\
\hline Jun 17.0 & $12.133 \pm 0.249$ & $4.220 \pm 0.120$ & $2.392 \pm 0.082$ & - & $\mathrm{CnB}$ \\
\hline Jun 26.0 & $12.189 \pm 0.522$ & $3.556 \pm 0.350$ & $1.903 \pm 0.548$ & - & $\mathrm{C}$ \\
\hline Jul 18.9 & $11.230 \pm 0.356$ & $2.510 \pm 0.161$ & $1.032 \pm 0.104$ & - & $\mathrm{C}$ \\
\hline Aug 11.8 & $8.687 \pm 0.341$ & $2.477 \pm 0.155$ & $1.084 \pm 0.091$ & - & $\mathrm{C}$ \\
\hline
\end{tabular}

Table 1. Very Large Array observations of SN 2009bb.

\begin{tabular}{cccc}
\hline \hline $\begin{array}{c}\text { Date } \\
(\mathrm{UT})\end{array}$ & $\begin{array}{c}\text { Frequency } \\
(\mathrm{MHz})\end{array}$ & $\begin{array}{c}\text { Flux Density } \\
(\mathrm{mJy})\end{array}$ & $\begin{array}{c}\text { Synthesized Beam Size } \\
(\operatorname{arcsec})\end{array}$ \\
\hline May 31.5 & 1287 & $4.6 \pm 0.3$ & $5 \times 2$ \\
Jun 10.6 & 617 & $3.0 \pm 0.3$ & $10 \mathrm{x} 4$ \\
Aug 8.3 & 617 & $7.9 \pm 0.3$ & $10 \mathrm{x} 4$ \\
Aug 10.4 & 332 & $3.3 \pm 0.7$ & $13 \times 7$ \\
\hline
\end{tabular}

Table 2. GMRT observations of SN 2009bb.

\begin{tabular}{cccc}
\hline \hline $\begin{array}{c}\text { Date } \\
(\mathrm{UT})\end{array}$ & $\begin{array}{c}\text { Exposure } \\
(\mathrm{sec})\end{array}$ & $\begin{array}{c}\text { Flux } \\
\left(\mathrm{erg} \mathrm{cm} \mathrm{cm}^{-2} \mathrm{~s}^{-1}\right)\end{array}$ & Mission \\
\hline Mar 24.1 & 3964 & $\lesssim 1.3 \times 10^{-13}$ & Swift/XRT \\
Apr 6.2 & 9829 & $\lesssim 1.7 \times 10^{-13}$ & Swift/XRT \\
Apr 10.5 & 6720 & $\lesssim 2.5 \times 10^{-13}$ & Swift/XRT \\
Apr 18.7 & 12752 & $\lesssim 3.0 \times 10^{-13}$ & Swift/XRT \\
Apr 18.6 & 9914 & $2.3 \pm 0.5 \times 10^{-14}$ & Chandra \\
\hline
\end{tabular}

Table 3. X-ray observations of SN 2009bb. All measurements are $0.3-10 \mathrm{keV}$ and unabsorbed. Upper limits are dominated by diffuse host galaxy emission. 


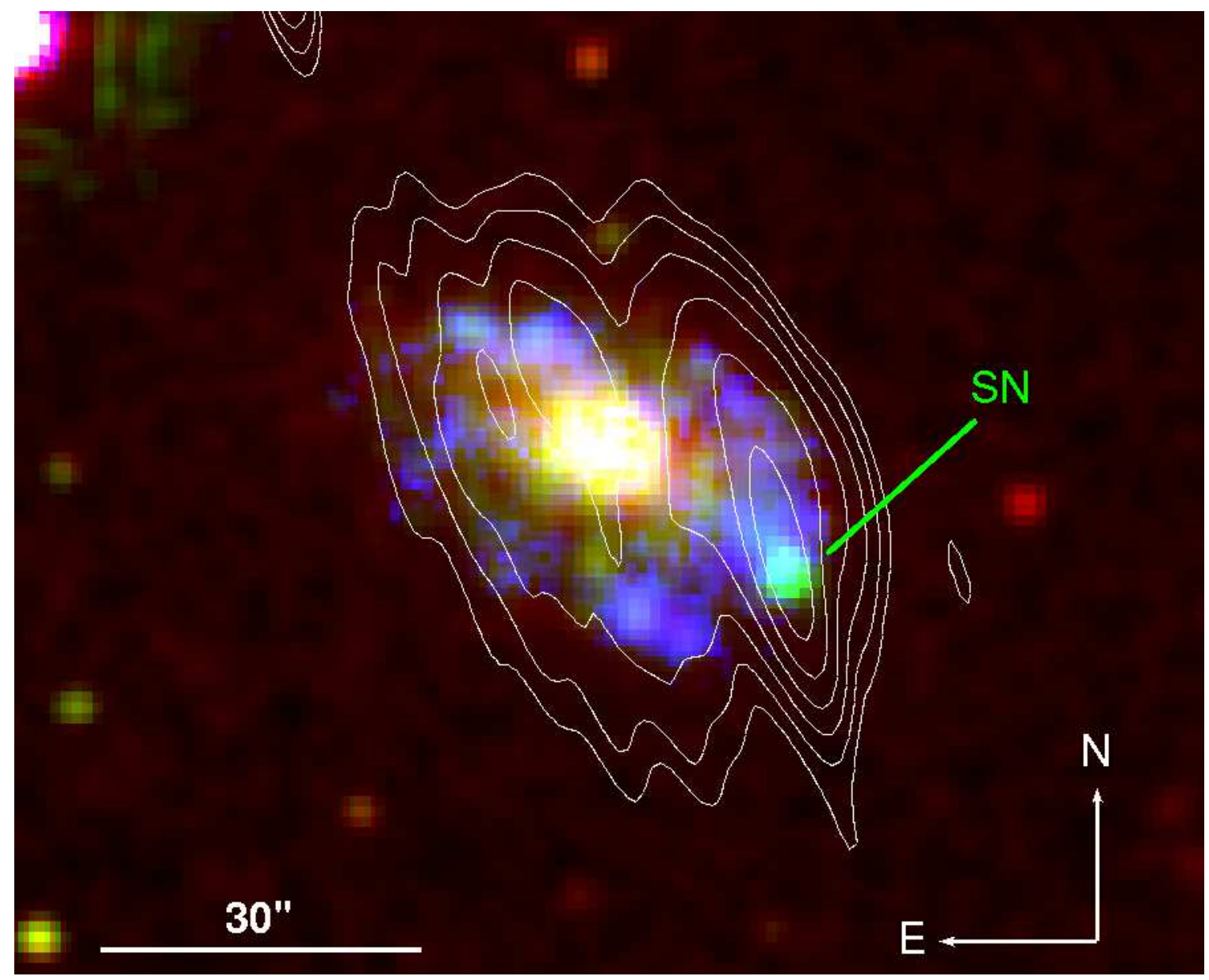

Figure 5. A composite image of the host galaxy of SN 2009bb, NGC 3278, constructed from 2MASS near-IR (red), Swift/UVOT optical (V-band, green) and UV (UVW2-filter, blue) images. Contours defining the structure of the diffuse radio $(1.43 \mathrm{GHz})$ emission as observed with the VLA in B-configuration are over-plotted and trace the regions of star-formation in the galaxy. The SN is clearly detected in the radio and optical images (labeled) and lies within a strongly star-forming region of the galaxy. 


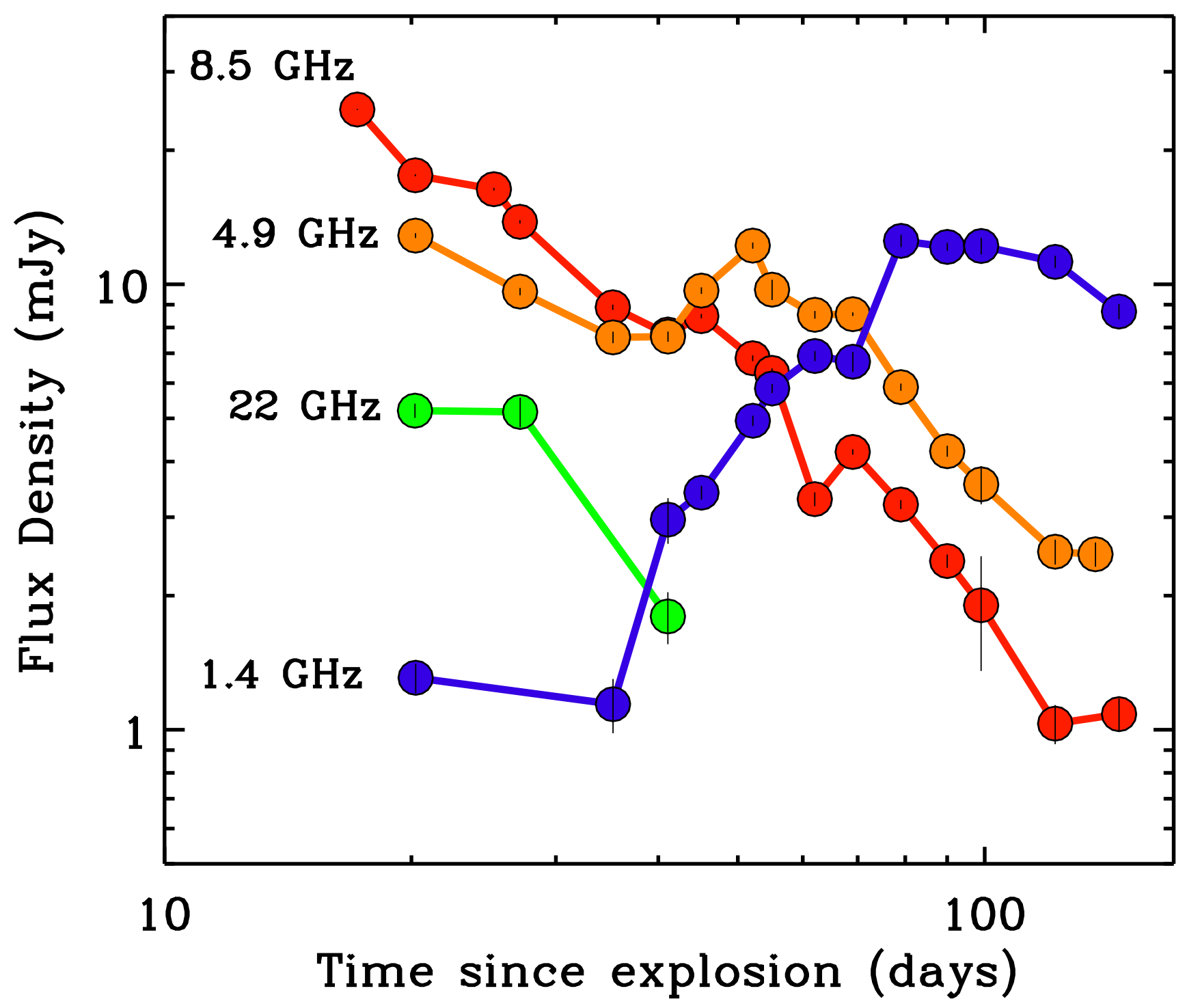

Figure 6. Radio light-curves for SN 2009bb at multiple frequencies. 